\title{
Deutschland in der ersten Globalisierung
}

Das Zeitalter der ersten Globalisierung beginnt 1870, eingefasst von der Öffnung der First Transcontinental Railroad im Mai 1869, der Öffnung des Suezkanals im November 1869 und der Gründung des Deutschen Kaiserreichs im Januar 1871. Die First Transcontinental Railroad schuf die erste durchgehende Eisenbahnverbindung zwischen der US-amerikanischen Ost- und Westküste. Die Öffnung des Suezkanals führte zum endgültigen Durchbruch der Dampfschifffahrt. Und im Verbund trugen Eisenbahnen und Dampfschiffe wesentlich dazu bei, dass die internationalen Transportkosten stetig sanken. ${ }^{1}$ Mit der Gründung des Deutschen Reichs 1871 wiederum war zumindest mit Blick auf die politischen Grenzen eine internationale Ordnung festgelegt, die bis 1914 weitgehend Bestand haben sollte.

Charakteristisch für diese erste Globalisierung war eine massive Ausweitung im internationalen Güterhandel und damit der räumlichen Trennung von Produktion und Verwendung. Im Gegensatz zu früheren Zeiten betraf dieser globale Handel Güter des alltäglichen Gebrauchs. Nicht nur Seide und seltene Gewürze, sondern einfache Textilien aus Baumwolle, Weizen und Roggen, Wein und Kaffee, Spielzeug aller Art aber auch Möbel und Maschinen wurden in zunehmenden Umfang rund um den Globus gehandelt, denn Produktion und Verwendung dieser Güter waren oft durch Tausende von Kilometern getrennt. ${ }^{2}$ Unternehmer und Konsumenten, aber auch Arbeitnehmer im Deutschen Reich profitierten teilweise enorm von dieser neuen globalen Arbeitsteilung. Andererseits waren sie dadurch auch vielfältigen und neuartigen Risiken ausgesetzt.

(C) Der/die Autor:in(nen) 2021. Open Access: Dieser Artikel wird unter der Creative Commons Namensnennung 4.0 International Lizenz veröffentlicht (creativecommons.org/licenses/by/4.0/deed.de).

Open Access wird durch die ZBW - Leibniz-Informationszentrum Wirtschaft gefördert.

1 Damit wurde es Ende 1869 möglich, in etwa 80 Tagen um die Welt zu reisen, was Jules Verne zu seinem bekannten Roman inspirierte.

2 Ein etwas ausführlicherer Überblick zum deutschen Außenhandel seit etwa 1850 bis 1914 ist bei Hungerland und Lampe (2021) zu finden.

Prof. Dr. Nikolaus Wolf lehrt Volkswirtschaftslehre und Wirtschaftsgeschichte an der HumboldtUniversität zu Berlin.

\section{Entwicklung des Außenhandels bis 1914}

Die Datengrundlage der folgenden Darstellung ist ein neuer umfassender Datensatz zum deutschen Außenhandel von Hungerland und Wolf (2021). Mit der Zolltarifreform des Jahres 1879 wurde die Außenhandelsstatistik des Deutschen Reichs neu geordnet und der Handel nach Handelspartnern und Gütergruppen deutlich detaillierter als jemals zuvor erfasst. ${ }^{3}$ Nach diesen Daten entwickelte sich der Außenhandel des Deutschen Kaiserreichs sehr dynamisch. Die Importe wuchsen pro Jahr um 3,7 \% (von 1,74 Mrd. Mark auf 5,73 Mrd. Mark), die Exporte um 3,2\% (von 1,87 auf 5,21 Mrd. Mark). Diese Zunahme im Außenhandel war durchaus bemerkenswert, denn der Anstieg war schneller als der Zuwachs im Bruttoinlandsprodukt (BIP), sodass der Offenheitsgrad der deutschen Volkswirtschaft (gemessen als Handel relativ zum BIP) deutlich zunahm. Der Wert der Importe relativ zum BIP stieg von 11\% (1880) auf $20 \%$ (1913), derjenige der Exporte im gleichen Zeitraum von $10 \%$ auf $18 \%$. Auch im Vergleich zu Großbritannien, der weltweit führenden Handelsmacht vor 1914, holte das Deutsche Reich rasant auf. Der Wert der Importe nahm von $42 \%$ (1880) auf $83 \%$ der britischen Importe (1913) zu (US-Dollar in Preisen von 1913), derjenige der Exporte wuchs sogar von $47 \%$ auf $95 \%$ (eigene Berechnungen nach Hungerland und Wolf, 2021; Federico und Tena-Junguito, 2019). Kurz vor dem Ersten Weltkrieg hatte sich das Deutsche Reich damit nicht nur zum industriellen Kernland des europäischen Kontinents entwickelt, sondern auch als eine der weltweit führenden Handelsmächte etabliert.

Die neue Datenbasis erlaubt einen detaillierten und zugleich systematischen Blick auf die Entwicklung der Handelspartner und Güter. Zunächst ist es sinnvoll, hier zwischen intensivem und extensivem Wachstum zu unterscheiden: Inwieweit war die Zunahme im Außenhandel von einem Wachstum im Handel in bestehenden Märkten mit schon bestehenden Partnern und Gütern (intensivem Wachstum) getrieben, inwiefern von der Erschließung neuer Märkte mit Blick auf Handelspartner und Güter (extensivem Wachstum)? Sowohl die Zahl der gehandelten Güter als auch die Zahl der Handelspartner nahm bis 1913 beträchtlich zu. Insgesamt

3 Ab 1880 wurden erstmals alle, auch die nicht zollpflichtigen, grenzüberschreitenden Warenströme erfasst, und es wurde neben deren Menge auch der Wert ermittelt. Weil Bremen und Hamburg erst 1888 dem deutschen Zollverein angeschlossen wurden, mussten für den neuen Datensatz von Hungerland und Wolf (2021) für die Jahre 1880 bis 1888 die Daten der Außenhandelsstatistik des deutschen Zollgebiets mit den tabellarischen Übersichten des Hamburgischen Handels und den Handelsdaten aus dem Jahrbuch für Bremische Statistik zusammengeführt und konsolidiert werden. 


\section{Abbildung 1}

Extensives und intensives Wachstum des deutschen Außenhandels, 1880 bis 1913

in \%

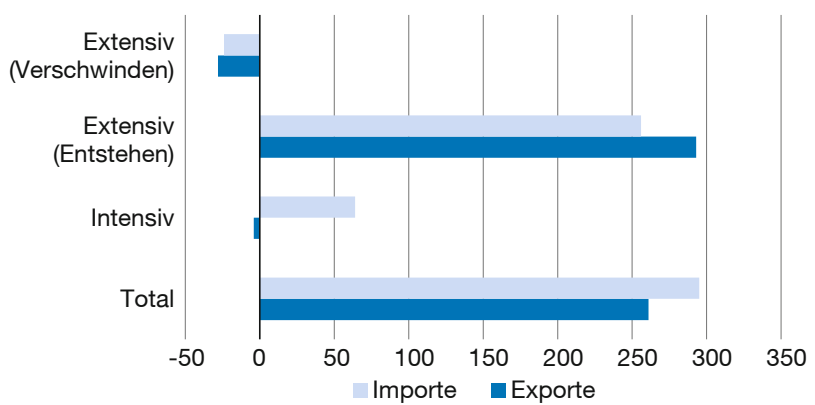

Quelle: Hungerland und Wolf (2021).

wurden 1880 noch 245 verschiedene Produktgruppen ${ }^{4}$ importiert und 248 exportiert, bis 1913 waren es 776, bzw. 782. Im gleichen Zeitraum stieg die Zahl der Länder, aus denen importiert wurde von 34 auf 85, die Zahl der Länder, in die von Deutschland aus exportiert wurde, stieg von 34 auf 86 (in den jeweiligen Grenzen). Die Abbildung 1 zeigt, wie sich damit das Wachstum des Außenhandels in extensives und intensives Wachstum zerlegen lässt.

Hinter dem beeindruckenden Außenhandelswachstum bis 1914 steckte also eine durchaus komplizierte Dynamik. Das Wachstum der Exporte war vollständig von der Eroberung neuer Märkte getrieben, wobei gleichzeitig immer auch kleinere Märkte schrumpften oder völlig verschwanden. Ähnlich aber etwas weniger ausgeprägt war diese Entwicklung bei den Importen. Hier war ein gewisser Teil des Wachstums tatsächlich intensiv, also auf eine Ausweitung schon bestehender Marktbeziehungen zurückzuführen. Ein prominentes Beispiel dafür ist der stark zunehmende Import von Getreide (vor allem Weizen) aus den USA nach Deutschland.

Insgesamt ist zu beobachten, dass sich das Deutsche Reich auf die Produktion von Industriegütern spezialisierte, während in zunehmenden Umfang Nahrungsmittel und Rohstoffe importiert wurden. Deutschland entwickelte, gemessen mit einem einfachen Lafay-Index, einen komparativen Vorteil bei Industriegütern, Maschinen und Chemischen Produkten (SITC Gruppen 5, 6 und 7) und einen komparativen Nachteil bei Nahrungsmitteln und Rohstoffen (SITC Gruppen 0 und 2). ${ }^{5}$ Allerdings war diese Entwicklung von einer zunehmenden Diversifizierung sowohl bei den Importen, als auch bei

4 Mit Produktgruppe ist hier eine sogenannte 5-Steller-Kategorie nach der Standard International Trade Classification - kurz SITC - (Revision 4) der Vereinten Nationen gemeint. Mehr zur Verwendung von SITC im Kontext historischer Statistik bei Hungerland und Altmeppen (2021).

5 Diese Gruppen beziehen sich wieder auf das SITC-System, diesmal die höchste Aggregationsstufe, die in zehn sektorale Gruppen gegliedert ist (FN 4). Zum Lafay-Index: Hungerland und Wolf (2021). den Exporten und von einem erstaunlich hohen Niveau von intra-industriellem Handel in allen Sektoren begleitet. Wichtiger noch: Schon vor 1914 war die „Industrialisierung“ des deutschen Außenhandels von einer starken Zunahme auch der industriellen Importe begleitet. Nach SITC-Gruppen gegliedert wuchsen nicht nur die Exporte sondern auch die Importe der Gütergruppen 5-7 deutlich überdurchschnittlich. Zudem wies das Deutsche Reich seit 1883 jedes Jahr ein Defizit im Güterhandel auf. Gliedert man die Handelsbilanz nach Sektoren und Handelspartnern, zeigt sich allerdings eine interessante Perspektive. Denn Deutschland erzielte im Außenhandel mit industriellen Gütergruppen einen stetig wachsenden Überschuss, dem ein ungefähr parallel wachsendes Handelsdefizit bei Nahrungsmitteln und Rohstoffen gegenüberstand. Und das Deutsche Reich erwirtschaftete diese Überschüsse vor allem im Handel mit seinen reichen europäischen Nachbarn (Frankreich, Großbritannien, Italien, den Niederlanden und Österreich-Ungarn). Hier scheint bereits die Grundlage für spätere Erfolge gelegt worden zu sein (Tilly und Kopsidis, 2020, 14).

\section{Ursachen und Folgen der Globalisierung}

Die Ursachen für diese Entwicklung können hier nur kurz angerissen werden und sind bisher auch nur unzureichend erforscht. Die Entwicklung passt zumindest zur Vorstellung der neoklassischen Außenhandelstheorie, nach der ein Land sich auf die Produktion solcher Güter spezialisieren wird, die besonders reichlich vorhandene Produktionsfaktoren intensiv nutzen. Im Vergleich zu vielen seiner Handelspartner war im Deutschen Reich Land relativ knapp, während Arbeit und Kapitel relativ reichlich vorhanden waren. Ein zunehmend gut organisierter Kapitalmarkt traf auf ein sehr reichlich vorhandenes Angebot an Arbeitskräften, die gleichzeitig immer besser ausgebildet waren. Im direkten Vergleich zu Großbritannien um 1900 arbeiteten deutsche Industriearbeiter mit vergleichbarer Produktivität zu niedrigeren Löhnen (Broadberry and Burhop, 2010). Eine wichtige Rolle spielte hier das weiterhin enorme Reservoir einer mobilen Landbevölkerung. Aber langfristig waren wohl die Fähigkeit der deutschen Börsen, Risikokapital bereitzustellen (Lehmann-Hasemeyer und Streb, 2016), ein leistungsfähiges und mit der Industrie vernetztes Bildungssystem und auch Deutschlands geografische Lage entscheidend für den Erfolg der ersten Globalisierung.

Welche gesamtwirtschaftlichen Folgen hatte die erste Globalisierung? Die Integration von Märkten bringt immer Gewinnende und Verlierende hervor, weil sie mit der Intensivierung von Wettbewerb einhergeht. Einen kausalen Zusammenhang zwischen dem Globalisierungsschock seit 1871 und wirtschaftlichem Strukturwandel stellen erstmals Bräuer et al. (2021) her. Sie zeigen, dass Landkreise, die besonders stark von der Produktion global gehandelter landwirtschaftlicher Produkte geprägt waren, in großem Umfang Beschäftig- 
te aber auch - durch Abwanderung - Bevölkerung verloren. Ein wesentlicher Teil dieser Menschen wanderte vermutlich in die wachsenden Städte und ihre Industrieunternehmen in anderen Teilen des Deutschen Reichs ab. Interessanterweise verhinderte diese Mobilität der Arbeitskräfte offenbar, dass es zu größeren Verlusten im Pro-Kopf-Einkommen oder Arbeitslosigkeit der betroffenen Regionen kam. Die Effekte der ersten Globalisierung auf regionale Unterschiede im ProKopf-Einkommen waren daher, ganz im Gegensatz zu heute, nahezu null.

Aber welche Wirkung hatte die erste Globalisierung auf das Niveau des Volkseinkommens und auf dessen Verteilung auf die Produktionsfaktoren Land, Kapital und Arbeit? Diese Frage ist empirisch schwer zu beantworten, weil Einkommen und Globalisierung zwar meist stark positiv korreliert sind, was aber nicht notwendig auf Kausalität schließen lässt. Lehmann und O'Rourke (2011) zeigen, dass zumindest die Liberalisierung von Agrarmärkten in Europa meist wachstumsfördernd wirkte. Nach O' Rourke (1997) führte die Grain Invasion von billigem Getreide aus den USA nach Europa zu sinkenden Erträgen aus Landbesitz, steigenden Kapitalerträgen, aber nur in einigen europäischen Ländern zu steigenden Reallöhnen: etwa in Großbritannien, nicht aber in Frankreich oder Schweden.

Pfister (2020) zeigt für das Deutsche Reich, dass das reale Nettonationalprodukt pro Kopf zwischen 1880 und 1913 um etwa 1,5\% pro Jahr wuchs. Der Anteil des Produktionsfaktors Land am Volkseinkommen sank in dieser Zeit deutlich, derjenige von Kapital dagegen nahm stark zu, während der Anteil des Faktors Arbeit etwa stabil blieb. Zudem stiegen seit 1890 die Reallöhne deutlich, insbesondere in den Industrieregionen des Deutschen Reichs (Pfister, 2018; Bartels et al., 2021). Inwieweit hier jedoch ein kausaler Zusammenhang zur ersten Globalisierung besteht, ist noch ungeklärt. Die Einkommensungleichheit, gemessen am Anteil der Top 1\% an allen Einkommen, nahm zwischen 1871 und 1900 deutlich zu und stagnierte dann in den eher industriell geprägten Regionen, während die Ungleichheit in den ländlichen Regionen weiter anstieg (Albers und Bartels, 2021; Bartels et al., 2021). Eine plausible Erklärung dafür ist, dass die globale Nachfrage nach deutschen Industrieprodukten zunächst zu stark ansteigenden Unternehmergewinnen führte, bevor zeitlich verzögert (und erkämpft durch zahlreiche Streiks und politische Zugewinne der Sozialdemokratie seit 1890) auch die Industriearbeiterschaft davon profitieren konnte. Interessanterweise scheinen die Reallohnsteigerungen den Exporterfolg der deutschen Industrie nicht geschmälert zu haben. Biermann (im Erscheinen) liefert allerdings Hinweise darauf, dass die Integration der Märkte zwischen 1875 und 1907 zu einer Konzentration hin zu größeren Unternehmen beigetragen haben könnte, die vermutlich auch überdurchschnittliche Löhne zahlten. Ein weiterer Aspekt betrifft die Vorteile, die Konsu- menten durch ein globales Angebot an Produkten haben. Wie dargestellt nahm die Zahl der gehandelten Produkte bis 1914 enorm zu. Hungerland (2017) zeigt, dass daraus beträchtliche Wohlfahrtsgewinne für Konsumenten resultierten, vor allem, weil Konsumenten damals stärker als heute auf Preisunterschiede für ähnliche Produkte reagierten.

\section{Reaktion von Wirtschaft und Politik auf die Globalisierung}

Die globale Integration der Gütermärkte seit 1870 übte also einen Anpassungsdruck aus, der zu Strukturwandel (sinkender Beschäftigung in der Landwirtschaft) und Migration führte und von steigender Nachfrage nach Kapital und Arbeitskraft gegenüber Land begleitet war. Aber wenngleich diese Entwicklungen von außen angestoßen worden waren, insbesondere durch fallende Transportkosten, versuchten Akteure in Wirtschaft und Politik doch, auf diese Globalisierung aktiv zu reagieren und sie zumindest teilweise mitzugestalten. Insbesondere die Zollpolitik hat in der Forschung viel Aufmerksamkeit erfahren. Preußen hatte seit den 1860er Jahren eine Politik des Freihandels verfolgt, der 1865 auch der Zollverein und zunächst das Deutsche Reich von 1871 folgte. In Sektoren, in denen sich ein komparativer Nachteil gegenüber Deutschlands Handelspartnern herausstellte, vor allem im Getreideanbau aber auch in der deutschen Schwerindustrie, die nach 1873 in eine Krise geraten war, bildeten sich seit Mitte der 1870er Jahre Lobbygruppen, die für Schutzzölle gegenüber der ausländischen Konkurrenz eintraten. Lehmann (2010) zeigt, dass sich die Verschiebung hin zu einer protektionistischen Mehrheit im Reichstag von 1878 zumindest teilweise durch wirtschaftliche Interessen der Wählerschaft erklären lassen. Allerdings spielten hier immer auch andere Faktoren, etwa strategische Interessen Bismarcks und der katholischen Zentrumspartei hinein. Im Rückblick hat die zollpolitische Wende von 1878/1879 die erste Globalisierung nur unwesentlich abgebremst. Zwar stiegen die Durchschnittszolläquivalente (Importzolleinnahmen als Anteil der Importwerte) von 1879 bis etwa 1900, sanken dann aber bis 1914, weil bei steigenden Preisen die spezifischen Zölle kaum angepasst wurden (Lampe und Sharp, 2013). Im europäischen Vergleich blieben die deutschen Zölle übrigens unter dem Durchschnitt, mit Ausnahme weniger Güter wie Getreide, Textilien, Eisen und Stahl (Hungerland und Lampe, 2021, Tabelle 1).

Durch die Zölle wurde der Strukturwandel keineswegs aufgehalten, auch weil zugleich die Transportkosten weiter sanken. Allerdings hatten die Zölle zum Teil beachtliche Verteilungseffekte, weil sie die Einkommen vor allem der Besitzer großer Güter stabilisierten, was über erhöhte Lebensmittelpreise vor allem die Konsumenten in den Städten finanzieren mussten (Wehler, 2006, 3, 650 ff). Der Fokus auf die Zollpolitik verstellt außerdem leicht den Blick auf andere Reaktionen 
auf die globale Marktintegration, die nicht weniger bedeutsam waren. Viele Kleinbauern etwa wurden durch den Verfall der Getreidepreise dazu bewegt, Produktions- und Kreditgenossenschaften zu gründen, um auf kapitalintensivere und weniger "globalisierte“ Produkte wie etwa Vieh- und Milchwirtschaft umzusteigen (Süsse und Wolf, 2020). Auch die Einführung der Sozialversicherungen seit 1883 ist vor dem Hintergrund der neuartigen Risiken zu sehen, denen Arbeitskräfte durch die Industrialisierung ausgesetzt waren. Obwohl diese Überlegungen für deren eigentliche Einführung kaum entscheidend waren, haben die Sozialversicherungen doch dazu beigetragen, die Kosten der ersten Globalisierung etwas gerechter zu verteilen (Jopp und Streb, 2021).

Mit den Schutzzollinteressen waren vor allem in der Schwerindustrie außerdem Bestrebungen zur Bildung von Kartellen verbunden, die vom Staat geduldet und teilweise gestützt wurden. Unter anderem wurde versucht, die durch Zölle erhöhten Preise im Inland für den Preiswettbewerb auf den Auslandsmärkten (in Form von Dumping) zu nutzen (Stolper et al., 1964, 55; Webb, 1980). Auch in der Landwirtschaft gab es mit dem Zuckerkartell und dem Spirituskartell Zusammenschlüsse, die von hohen Importzöllen profitierten (Roelevink und Ziegler, 2021). Insgesamt sollten diese interventionistischen Tendenzen zu Schutzzoll und Kartellen im Kaiserreich jedoch nicht überbetont werden. Die Schutzzölle spielten empirisch keine bedeutende Rolle für die wirtschaftliche Dynamik vor 1914. Die globale Verflechtung der deutschen Wirtschaft nahm bis 1914 stetig zu, zum Vorteil der großen Mehrheit der Unternehmen, Arbeiter und Konsumenten.

\section{Lehren für heute?}

Kann man aus der historischen Erfahrung der ersten Globalisierung Lehren für heute ziehen? Zunächst: Zölle waren vor 1914 keine sinnvolle Antwort und sind es heute sicherlich auch nicht. Die Verflechtung der deutschen Wirtschaft hat in den vergangenen Jahrzehnten weiter zugenommen. Deutschlands Exporterfolge sind bis heute von den Sektoren geprägt, die schon vor 1914 eine positive Handelsbilanz auswiesen. Und weiterhin erwirtschaftet Deutschland knapp die Hälfte des Außenhandelsüberschusses im Handel mit EU-Staaten. ${ }^{6}$ Aber was sich schon vor 1914 angebahnt hatte, gilt heute noch viel mehr: dass nämlich industrielle Exporte mit Importen einhergehen. Nicht nur Produktion und Verwendung, sondern die Produktion selbst findet heute in Form von fragmentierten Wertschöpfungsketten in unterschiedlichen Ländern statt. „Deutsche“ Exporte sind oft das Ergebnis internationaler (besonders häufig europäischer)

6 Außerdem erzielt Deutschland heute einen beträchtlichen Außenhandelsüberschuss mit den USA (ganz anders als vor 1914), während die deutsche Handelsbilanz mit China (und den meisten Staaten in Ostund Südostasien) stark negativ ist.
Produktion. Mehr als früher hängt Deutschlands Außenhandelserfolg also vom Wohlstand seiner Nachbarn ab. Zudem wird Deutschlands Außenhandel heute stärker als früher von wenigen Branchen getragen, was neue Risiken mit sich bringt. Allein der Export von Kraftfahrzeugen macht heute mehr als $15 \%$ aller deutschen Exporte aus. Es besteht damit zum einen das Risiko, dass nur wenige Unternehmen vom Außenhandel profitieren; zum anderen können durch diese Konzentration marktspezifische Risiken enorme gesamtwirtschaftliche Probleme mit sich bringen. Die hohe und zunehmende Diversifizierung des deutschen Außenhandels vor 1914 war vorteilhaft.

Die erste Globalisierung ging mit einem umfassenden Strukturwandel von einer agrarischen hin zu einer industriell geprägten Wirtschaft einher. Die Anpassung erfolgte weitgehend über Abwanderung von Arbeitskräften aus schrumpfenden ländlichen Regionen in wachsende Städte (und bis etwa 1900 auch nach Übersee). Davon profitierten jedoch nicht nur die „Kapitalbesitzer“, also Unternehmer und Investoren, sondern auch die Arbeitnehmer, die sich seit 1890 durch deutlich steigende Reallöhne für ihre Mobilität entschädigt sahen. Entscheidend war, dass durch die steigende Nachfrage nach deutschen Industrieprodukten vor 1914 auch die Nachfrage nach Arbeitskraft stieg.

Dagegen trifft die Globalisierung von heute in Deutschland auf eine etablierte Industrielandschaft, und Deutschland steht im Wettbewerb mit zahlreichen anderen Industrieländern. Von einer weiteren Vertiefung der Marktintegration profitieren tendenziell Kapitalbesitzer und hochqualifizierte Arbeitnehmer. Gerade durch die globale Fragmentierung der Produktion (und durch institutionelle Änderungen) ist die Verhandlungsposition der Arbeitnehmer insgesamt geschwächt, was sich in stagnierenden Reallöhnen und zugleich wachsender Einkommensungleichheit widerspiegelt. Eine deutlich niedrigere Mobilität führt außerdem dazu, dass spezifische sektorale Schocks stärker als vor 1914 zu regionalen Einkommensunterschieden und Arbeitslosigkeit beitragen. Um diesem hohen Anpassungsdruck auf dem Arbeitsmarkt standhalten zu können, bedarf es einerseits mehr denn je Innovation und besserer Bildung, denn nur gute Qualifikation und hohe Arbeitsproduktivität kann Arbeitsplätze in Deutschland sichern. Zugleich sollte heute über grenzüberschreitende institutionelle Absicherungen für Arbeitnehmer nachgedacht werden, um die - vor allem durch technologische Entwicklungen - im historischen Vergleich dramatisch geschwächte Verhandlungsposition der Arbeitnehmer zu stärken. Im Vergleich zur ersten Globalisierung vor 1914 ist es sicherlich schwieriger geworden, zugleich international wettbewerbsfähig zu bleiben und eine gerechte Verteilung der Globalisierungsgewinne zu sichern. Langfristig wird beides gelingen müssen, damit Deutschland auch von der zweiten Globalisierung profitieren kann. 


\section{Literatur}

Albers, T. N. H. und C. Bartels (2021), Phasen und Triebkräfte der Ungleichheit in Deutschland von 1840 bis 1914, in U. Pfister et al. (Hrsg.), Deutschland 1871. Die Nationalstaatsbildung und der Weg in die moderne Wirtschaft, 291-312.

Bartels, C., F. Kersting und N. Wolf (2021), Testing Marx. Income Inequality, Concentration, and Socialism in late 19th century Germany, EHES Working Paper, 211, European Historical Economics Society (EHES).

Biermann, M. (im Erscheinen), Trade and the size distribution of firms: Evidence from the German Empire, German Economic Review.

Bräuer, R., W.-F. Hungerland und F. Kersting (2021), Trade Shocks, Labor Markets and Elections in the First Globalization, Humboldt Universität zu Berlin.

Broadberry, S. und C. Burhop (2010), Real Wages and Labor Productivity in Britain and Germany, 1871-1938: A Unified Approach to the International Comparison of Living Standards, The Journal of Economic History, 70(2), 400-427.

Federico, G. und A. Tena-Junguito (2019), World trade, 1800-1938: a new synthesis, Revista de Historia Económica - Journal of Iberian and Latin American Economic History, 37(1).

Hungerland, W.-F. (2017), The Gains from Import Variety in Two Globalisations: Evidence from Germany, EHES Working Paper, 120, European Historical Economics Society (EHES).

Hungerland, W.-F. und C. Altmeppen (2021), What is a product anyway? Applying the Standard International Trade Classification (SITC) to historical data, Historical Methods: A Journal of Quantitative and Interdisciplinary History, 1(15), 1-15.

Hungerland, W.-F. und M. Lampe (2021), Globalisierung und Außenhandel, in U. Pfister et al. (Hrsg.), Deutschland 1871. Die Nationalstaatsbildung und der Weg in die moderne Wirtschaft, 335-360.

Hungerland, W.-F. und N. Wolf (2021), The Panopticon of Germany's Foreign Trade, 1880 - 1913. New facts on the First Globalization, Humboldt Universität zu Berlin.

Jopp, T. und J. Streb (2021), Die Einführung der Bismarckschen Sozialversicherung und ihre Folgen für die Arbeits- und Lebensverhältnisse, in U. Pfister, J.-O. Hesse, M. Spoerer und N. Wolf (Hrsg.), Deutschland 1871. Die Nationalstaatsbildung und der Weg in die moderne Wirtschaft, 267-289.
Lampe, M. und P. Sharp (2013), Tariffs and Income. A Time series analysis for 24 Countries, Cliometrica, 7(2), 207-235.

Lehmann, S. (2010), The German Elections in the 1870s. Why Germany turned from Liberalism to Protectionism, The Journal of Economic History, 70(1), 146-178.

Lehmann, S. und K. H. O' Rourke (2011), The Structure of Protection and Growth in the late Nineteenth Century, Review of Economics and Statistics, 93(2), 606-616.

Lehmann-Hasemeyer, S. und J. Streb (2016), The Berlin Stock Exchange in Imperial Germany - A Market for New Technology?, American Economic Review, 106(11), 3558-3576.

O' Rourke, K. (1997), The European Grain Invasion, 1870-1913, The Journal of Economic History, 57(4), 775-801.

Pfister, U. (2018), Real Wages in Germany during the First Phase of Industrialization, 1850-1889, Jahrbuch für Wirtschaftsgeschichte, 59(2), 567-596.

Pfister, U. (2020), The Crafts-Harley View of German Industrialization. An Independent Estimate of the Income Side of net National Product, 1851-1913, European Review of Economic History, 24(3), 502-521.

Roelevink, E.-M. und D. Ziegler (2021), Wie organisiert war der Kapitalismus im Kaiserreich? Verbände, Kartelle und personelle Verflechtungen, in U. Pfister, J.-O. Hesse, M. Spoerer und N. Wolf (Hrsg.), Deutschland 1871. Die Nationalstaatsbildung und der Weg in die moderne Wirtschaft, 245-266.

Süsse, M. und N. Wolf (2020), Rural Transformation, Inequality and the Origins of Micro-Finance, Journal of Development Economics, 143 (März).

Stolper, G., K. Häuser und K. Borchardt (1965), Deutsche Wirtschaft seit 1870, Mohr.

Tilly, R. H. und M. Kopsidis (2020), From Old Regime to Industrial State. A history of German Industrialization from the Eighteenth Century to World War I, University of Chicago Press.

Webb, S. B. (1980), Tariffs, Cartels, Technology and Growth in the German Steel Industry, 1879 to 1914, The Journal of Economic History, 40(2), 309-329.

Wehler, H.-U. (2006), Deutsche Gesellschaftsgeschichte, Bd. 3, Von der "Deutschen Doppelrevolution“ bis zum Beginn des Ersten Weltkrieges, 1849-1914, C.H. Beck. 